\title{
Home visits providing diet advice may reduce early childhood caries
}

\author{
Do home visits that provide new mothers with advice about breastfeeding and \\ weaning reduce the odds of infants developing early childhood caries?
}

Feldens CA, Vitolo MR, Drachler Mde L.

A randomised trial of the effectiveness of home visits in preventing early childhood caries. Community Dent Oral Epidemiol 2007; 35:215-223

Design This was a randomised controlled trial (RCT).

Intervention Participants were randomised to either the control group, which received routine assistance from health service paediatricians, or an intervention group that received these routine visits and, in addition, regular home visits from trained fieldworkers who advised on healthy breastfeeding and weaning practice based on World Health Organization recommendations.

Outcome measure The prevalence of early childhood caries (ECC) was assessed by visual inspection at 12 months and the odds ratio (OR) of developing ECC at 12 months was calculated. ECC was considered present if one or more decayed surfaces were detected.

Results ECC was recorded in $10.2 \%$ of the intervention group and $18.3 \%$ of the control group. The odds of developing ECC was $48 \%$ lower for the intervention group than in the control group when adjusted for number of teeth [OR, 0.52; $95 \%$ confidence interval $(\mathrm{Cl})$, $0.27-0.97]$. A significant difference in the mean number of decayed surfaces was found between the groups [intervention group, 0.37; standard deviation (SD), 1.37; control group, 0.63; SD, 1.62; Mann Whitney $U$ test P 0.03].

Conclusions Home visits giving dietary advice appear to help reduce ECC in infants.
Address for correspondence: CA Feldens, Universidade Luterana do Brasil - Curso de Odontologia, Av. Farroupilha 8001, Prédio 59, Bairro São José, Canoas RS 92425-900, Brazil. E-mail: feldens@brturbo.com.br

\section{Commentary}

ECC is a public health problem in both the developed and developing world particularly, for those living in disadvantaged communities. ${ }^{1}$ It has been argued that, to maximise improvements in oral health, people involved in the promotion of oral health should adopt a common risk factor approach rather than tackling oral diseases specifically. ${ }^{2}$ This approach is tested in this RCT, which is itself part of a much larger study assessing the effect of home visits in which mothers are advised about the impact of breastfeeding and weaning on children's feeding practices and general health.

Mothers who gave birth within the public health system in a city in Southern Brazil, a population generally from low socio-economic backgrounds, were assigned to control $(n=300)$ or intervention $(n=200)$ groups using block randomisation. A power calculation was performed although this was based on detecting a very large $(60 \%)$ difference in the odds of developing dental caries at the age of 1 year. The study in fact only detected a difference of $48 \%$ in the OR of developing ECC by that age. This may mean that further followup studies, which the authors anticipate undertaking, are compromised by the initial sample size.

Although no stratification of the population was undertaken, both groups seemed to be balanced in terms of socio-economic indicators recognised as predictors of ECC experience. No information was reported, however, about any differences in oral hygiene practices between the two groups, which could be a confounding factor. Also, as with many community-based studies, there is the potential for cross-contamination of the control group, with mothers from the intervention group advising those in the control group of the additional information they received. Such cross-contamination can mask the true effect of the intervention. It is possible to reduce this potential bias by stratifying the control and intervention groups by geographical area. Participants in the study could not be masked to their intervention but the dentist undertaking the dental examination was masked to the participants' intervention group, thus helping to reduce bias.

The definition of dental decay used was "any white spot lesion or cavity", which may have led to overdiagnosis of dental decay because of other causes of white spot lesions. The study does report a high degree of intra-examiner reproducibility, suggesting that examinations were conducted in a consistent manner.

The reported OR of developing dental caries is 0.52 , meaning that the chance of developing ECC was $48 \%$ lower in the intervention group. Although this is statistically significant, the $95 \% \mathrm{CI}$ is very wide, ranging from $0.27-0.97$.

Although the authors report an intention to treat analysis, only results from participants who received the dental examination at 12 months were included in the final analysis. Calculating 
the result based on the those originally allocated to the test and control groups, the odds of developing ECC would have been 0.60 (with 95\% CI ranging from 0.35-1.03) with a numberneeded-to-treat to render one additional child free of ECC of 19 (95\%CI, 9-2187).

The study also details the reported feeding practices of the two groups, which may be relevant in the development of ECC. The intervention group reported undertaking more of the positive feeding behaviours than the control group some detail of what these were would be interesting here and elsewhere, although this may be subject to reporting bias.

This RCT finds evidence that the provision of intensive advice on breastfeeding and weaning practice does improve the oral health of children at 1 year of age. The results of the study must be interpreted with caution, nevertheless, because of those issues raised above.
Undertaking a larger study, perhaps looking at longer-term outcomes, may provide more conclusive evidence of the benefits of such an approach on oral health.

\section{Cameron Walker}

Glasgow Dental Hospital and School, Glasgow

Valerie AA White

Department of Dental Public Health, NHS Fife and Lothian, Edinburgh, Scotland, UK

1. Davies GN. Early childhood caries - a synopsis. Community Dent Oral Epidemiol 1998; 26 (suppl. 1): S106-S116.

2. Sheiham A, Watt RG. The Common Risk Factor Approach: a rational basis for promoting oral health. Community Dent Oral Epidemiol 2000; 28:399-406.

Evidence-Based Dentistry (2007) 8, 108-109. doi:10.1038/sj.ebd.6400527 